\title{
Development of Net-Current Free Heliotron Plasmas in the Large Helical Device
}

A.Komori 1), H.Yamada 1), O.Kaneko 1), K.Kawahata 1), T.Mutoh 1), N.Ohyabu1 ), S.Imagawa 1), K.Ida 1), Y.Nagayama 1), T.Shimozuma 1), K.Y.Watanabe 1), T.Mito 1), M.Kobayashi 1), K.Nagaoka 1), R.Sakamoto 1), N.Yoshida 2), S.Ohdachi 1), S.Sakakibara 1), N.Ashikawa 1), Y.Feng 3), T.Fukuda 4), H.Igami 1), S.Inagaki 2), H.Kasahara 1), S.Kubo 1), R.Kumazawa 1), O.Mitarai 5), S.Murakami 6), Yuji Nakamura 7), M.Nishiura 1), T.Hino 8), S.Masuzaki 1), K.Tanaka 1), K.Toi 1), A.Weller 3), M.Yoshinuma 1), Y.Narushima 1), N.Ohno 9), T.Okamura 10), N.Tamura 1), K.Saito 1), T.Seki 1), S.Sudo 1), H.Tanaka 1), T.Tokuzawa 1), N.Yanagi 1), M.Yokoyama 1), Y.Yoshimura 1), T.Akiyama 1), H.Chikaraishi 1), M.Chowdhuri 11), M.Emoto 1), N.Ezumi 12), H.Funaba 1), L.Garcia 13), P.Goncharov 1), M.Goto 1), K.Ichiguchi 1), M.Ichimura 14), H.Idei 2), T.Ido 1), S.Iio 10), K.Ikeda 1), M.Irie 15), A.Isayama 16), T.Ishigooka 17), M.Isobe 1), T.Ito 9), K.Itoh 1), A.Iwamae 16), S.Hamaguchi 1), K.Hamajima 18), S.Kitajima 18), S.Kado 19), D.Kato 1), T.Kato 1), S.Kobayashi 7), K.Kondo 7), S.Masamune 20), H.Matsumoto 8), N.Matsunami 21), T.Minami 1), C.Michael 1), H.Miura 1), J.Miyazawa 1), N.Mizuguchi 1), T.Morisaki 1), S.Morita 1), G.Motojima 1), I.Murakami 1), S.Muto 1), K.Nagasaki 7), N.Nakajima 1), Yukio Nakamura 1), H.Nakanishi 1), H.Nakano 1), K.Narihara 1), A.Nishimura 1), H.Nishimura 22), K.Nishimura 1), S.Nishimura 1), N.Nishino 23), T.Notake 1), T.Obana 1), K.Ogawa 9), Y.Oka 1), T.Ohishi 9), H.Okada 7), K.Okuno 24), K.Ono 25), M.Osakabe 1), T.Osako 26), T.Ozaki 1), B.J.Peterson 1), H.Sakaue 1), M.Sasao 19), S.Satake 1), K.Sato 1), M.Sato 1), A.Shimizu 1), M.Shiratani 2), M.Shoji 1), H.Sugama 1), C.Suzuki 1), Y.Suzuki 1), K.Takahata 1), H.Takahashi 1), Y.Takase 26), Y.Takeiri 1), H.Takenaga 16), S.Toda 1), Y.Todo 1), M.Tokitani 1), H.Tsuchiya 1), K.Tsumori 1), S.Urano 16), E.Veshchev 1), F.Watanabe 9), T.Watanabe 1), T.H.Watanabe 1), I.Yamada 1), S.Yamada 1), O.Yamagishi 1), S.Yamaguchi 27), S.Yoshimura 1), T.Yoshinaga 1), O.Motojima 1)

1) National Institute for Fusion Science, Toki, Japan

2) Research Institute of Applied Mechanics, Kyushu University, Kasuga, Fukuoka, Japan

3) Max-Planck-Institut für Plasmaphysik, IPP-EURATOM-Association, Greifswald, Germany

4) Center for Atomic and Molecular Technologies, Osaka University, Suita, Osaka, Japan

5) Liberal Arts Education Center, Tokai University, Toroku, Kumamoto, Japan

6) Graduate School of Engineering, Kyoto University, Kyoto, Japan

7) Institute of Advanced Energy, Kyoto University, Uji, Kyoto, Japan

8) Graduate School of Engineering, Hokkaido University, Sapporo, Hokkaido, Japan

9) Graduate School of Engineering, Nagoya University, Nagoya, Aichi, Japan

10) Graduate School of Science and Engineering, Tokyo Institute of Technology, Tokyo, Japan

11) School of Physical Science, The Graduate University for Advanced Studies, SOKENDAI, Hayama, Kanagawa, Japan

12) Nagano National College of Technology, Nagano, Japan

13) Universidat Carlos III, Madrid, Spain

14) Plasma Research Center, Tsukuba University, Tsukuba, Ibaraki, Japan

15) School of Science and Engineering, Waseda University, Tokyo, Japan

16) Naka Fusion Institute, Japan Atomic Energy Agency, Naka, Ibaraki, Japan

17) Faculty of Science and Technology, Seikei University, Musashino, Tokyo, Japan

18) Graduate School of Engineering, Tohoku University, Sendai, Japan

19) Graduate School of Engineering, The University of Tokyo, Tokyo, Japan

20) Graduate School of Science and Technology, Kyoto Institute of Technology, Kyoto, Japan

21) EcoTopia Science Institute, Nagoya University, Nagoya, Aichi, Japan

22) Institute of Laser Engineering, Osaka University, Suita, Osaka, Japan

23) Graduate School of Engineering, Hiroshima University, Higashihiroshima, Hiroshima, Japan

24) Radiochemistry Research Lab., Shizuoka University, Shizuoka, Japan

25) Interdisciplinary Faculty of Science and Engineering, Shimane University, Matsue, Shimane, Japan

26) Graduate School of Frontier Science, The University of Tokyo, Kashiwa, Chiba, Japan

27) Organization for Research and Development of Innovative Science and Technology, KansaiUniversity, Suita, Osaka, Japan

e-mail contact of main author : komori@LHD.nifs.ac.jp 
Abstract Remarkable progress in the physical parameters of net-current free plasmas has been made in the Large Helical Device (LHD) since the last Fusion Energy Conference in Chengdu, 2006 (O.Motojima et al., Nucl. Fusion 47 (2007) S668). The beta value reached $5 \%$ and a high beta state beyond $4.5 \%$ from the diamagnetic measurement has been maintained for longer than 100 times the energy confinement time. The density and temperature regimes also have been extended. The central density has exceeded $1.0 \times 10^{21} \mathrm{~m}^{-3}$ due to the formation of an Internal Diffusion Barrier (IDB). The ion temperature has reached $6.8 \mathrm{keV}$ at the density of $2 \times 10^{19} \mathrm{~m}^{-3}$, which is associated with the suppression of ion heat conduction loss. Although these parameters have been obtained in separated discharges, each fusion-reactor relevant parameter has elucidated the potential of net-current free heliotron plasmas. Diversified studies in recent LHD experiments are reviewed in this paper.

\section{Introduction}

The Large Helical Device (LHD) is a large-scale heliotron device with a pair of helical coils and an intrinsic divertor [1]. It employs the largest superconducting magnets in the world which provides the confinement magnetic field in completely steady state [2]. The major goal of the LHD experiment is to demonstrate the high performance of helical plasmas in a reactor relevant plasma regime. Thorough exploration of net current free plasmas further demonstrates its potential as a fusion energy reactor. The intrinsic advantage that plasma current is not required has been demonstrated in better capability of steady-state and high-density operation than in tokamaks. Key issues in confinement physics of net current free plasmas have been identified, and commonalities and differences with tokamak physics can be discussed in detail. In particular with regard to three-dimensional effects, pioneering works validated in LHD are applicable to tokamaks.

LHD has a nominal plasma volume of $30 \mathrm{~m}^{3}$ with the major and minor radii of $3.7 \mathrm{~m}$ and 0.64 $\mathrm{m}$, respectively. The heating capability of neutral beam injection (NBI) has been significantly upgraded in these two years. A new perpendicular NBI with a low accelerating voltage of 40 $\mathrm{keV}(7 \mathrm{MW})$ enables efficient ion heating. Confinement of trapped particle used to be a great concern and indeed this is the reason why the tangential NBI was chosen at the beginning of the LHD project. However, this issue has been resolved by geometrical optimization [3] and demonstration of the confinement of highly energetic trapped particles up to $1.6 \mathrm{MeV}$ in an ICRF experiment [4] has motivated the choice of perpendicular NBI. Combined with the three existing tangential beams $(180 \mathrm{keV})$, the heating capability of the NBI has exceeded $20 \mathrm{MW}$. The upper limit of the magnetic field is improved by adding the subcooling system [5] to lower the inlet liquid helium temperature from $4.4 \mathrm{~K}$ to $3.2 \mathrm{~K}$. The increase in magnetic field is certainly small (4\%) but very beneficial to the resonance condition of the $\mathrm{ECH}$.

The plasma parameters as well as physical understanding have been progressing steadily since the last IAEA Fusion Energy Conference in 2006 [6]. The highlighted achievement in the latest experimental campaigns is the extension of high ion temperature [7], high $\beta$ [8] and high density [9]. Ion temperature has increased to $6.8 \mathrm{keV}$ due to the perpendicular NBI. In addition, this increase is associated with confinement improvements which share observation in common with internal transport barriers (ITB) in tokamaks. Also this high ion temperature regime is accompanied by a large toroidal flow [10] and an extremely hollow impurity profile which is called "Impurity Hole"[11]. 
The $\beta$ value has reached $5 \%$ which is the demanding target required for a fusion reactor and it should be emphasized that this high $\beta$ state is maintained for more than 100 times the energy confinement time. The innovative scenario of high density by means of an Internal Diffusion Barrier (IDB) [12], which was discovered in the Local Island Divertor configuration, has been extended to a helical divertor configuration [13]. The central density has exceeded $1 \times 10^{20} \mathrm{~m}^{-3}$ at the moderate magnetic field of $2.5 \mathrm{~T}$.

In addition to these major achievements, important progress has been made in various areas in these two years. Properties of anomalous transport have been discussed with respect to the effect of magnetic shear [14], dependence on collisionality [15] and long radial correlation [16]. Wave physics in Electron Bernstein wave heating [17], electron cyclotron current drive [18] and high harmonic fast wave heating [19] has been investigated. The physics of energetic particles has been discussed with emphasis on its radial profile [20] and TAE mode [21,22]. LHD has provided a good platform for the study of PWI. Co-deposition [23], behavior of dust [24] and damage on in-vessel mirrors [25] have been evaluated. These results are reported in individual contributions to this 22nd IAEA Fusion Energy Conference in Geneva, 2008.

\section{Extension of Operational Regime}

The synergism of technological upgrades and physical findings has extended the plasma parameters. In this section, the latest parameter extension is summarized before describing each topical result. Plasma parameters in representative discharges are described in Table 1.

The ion temperature is raised to $6.8 \mathrm{keV}$ at the moderate density of $2 \times 10^{19} \mathrm{~m}^{-3}$ [7]. In an earlier study, a high ion temperature as high as $13.5 \mathrm{keV}$ was realized by applying a heavier gas, argon, instead of hydrogen [26]. This scenario was attributed to the enhancement of ion heating under the condition of a tangential NBI energy as high as $180 \mathrm{keV}$ which primarily heats electrons in hydrogen operation. While this operation was limited to a very low density of $0.35 \times 10^{19} \mathrm{~m}^{-3}$, it validated the characteristic that the increase in input power to ions leads to the increase in ion temperature. Concern about the confinement of highly energetic trapped particles due to helical ripples was resolved by geometrical optimization [3]. A new perpendicular NBI with a lower energy $(40 \mathrm{keV})$ is favorable to ion heating. This beam works

TABLE I ACHIEVED PARAMETERS IN LHD SINCE THE LAST IAEA-FEC 2006

\begin{tabular}{|c|c|c|c|c|c|}
\hline & $\begin{array}{c}\text { high } \\
T_{i}\end{array}$ & $\begin{array}{c}\text { high } \\
\beta\end{array}$ & $\begin{array}{c}\text { High } \\
n_{e}\end{array}$ & $\begin{array}{l}\text { high } \\
n \tau_{\mathrm{E}} T\end{array}$ & $\begin{array}{c}\text { High } \\
W_{p}\end{array}$ \\
\hline$T_{e}(0) \quad(\mathrm{keV})$ & 3.5 & 0.4 & 0.3 & 0.55 & 1.1 \\
\hline$(\mathrm{keV})$ & 6.8 & - & 0.3 & 0.55 & - \\
\hline$n_{e}(0)\left(10^{19} \mathrm{~m}^{-3}\right)$ & 1.73 & 2.8 & 108 & 40 & 20 \\
\hline $\bar{n}_{e} \quad\left(10^{19} \mathrm{~m}^{-3}\right)$ & 2.05 & 3.0 & 41 & 20 & 13 \\
\hline (s) & 0.057 & 0.017 & 0.06 & 0.23 & 0.12 \\
\hline$(\%)$ & 0.93 & 5.0 & 1.37 & 0.85 & 1.56 \\
\hline$(\mathrm{kJ})$ & 884 & 90 & 762 & 779 & 1625 \\
\hline$(\mathrm{MW})$ & 15.8 & 5.4 & 13.3 & 2.92 & 14.4 \\
\hline (m) & 3.575 & 3.6 & 3.95 & 3.75 & 3.63 \\
\hline$(\mathrm{T})$ & 2.77 & 0.425 & 2.51 & 2.64 & 2.83 \\
\hline Remarks & $\begin{array}{c}\text { Hydrogen } \\
\text { dominant }\end{array}$ & $A_{p}=6.6$ & $\begin{array}{c}\text { IDB with } \\
\text { helical } \\
\text { divertor }\end{array}$ & $\begin{array}{c}\text { IDB with } \\
\text { helical } \\
\text { divertor }\end{array}$ & \\
\hline
\end{tabular}


as a diagnostics beam for the charge exchange spectroscopy which provides profiles of ion temperature and flow velocity, and consequently radial electric field. The experimental results will be discussed in Sec.3.

Tolerance against the interchange modes which have been observed in earlier works [27] can be maintained in the higher aspect ratio configuration with worse stability but better confinement and power deposition. Optimization of the compromise between confinement and MHD stability has led to a $\beta$ of $5 \%$. The scenario of an IDB has been applied to a high $\beta$ study at low magnetic field. The central $\beta$ reaches $10 \%$ in this case [8]. This study will be discussed in Sec.4.

The IDB regime has been extended significantly since plasma with an IDB has been reproduced in a helical divertor [13]. The local accumulation of neutrals can be avoided by the outward shift of the magnetic axis. Under this condition, the very high central density with an IDB has been realized without the efficient pumping of the LID. An impurity screening effect in the peripheral ergodic layers aids this high density operation [28]. The highest $n \tau_{\mathrm{E}} T$ of $5 \times 19 \mathrm{~m}^{-3} \cdot \mathrm{s} \cdot \mathrm{keV}$ is obtained by this scenario. The characterization of plasmas with IDB [9] will be described in Sec.5.

A new regime of the long pulse was examined exceeding the previous power limit by replacing divertor plates with new ones having better heat conductivity, which led to the extension of the plasma pulse length to $300 \mathrm{~s}$ by RF heating power of $1 \mathrm{MW}$. Then a central electron temperature and a central density are $1.5 \mathrm{keV}$ and $0.6 \times 10^{19} \mathrm{~m}^{-3}$, respectively. This subject [29] will be discussed in Sec.6.

\section{High Ion Temperature}

Improvement of ion heat transport in Heliotron plasmas has been realized by an upgrade of the ion heating power by using a low energy perpendicular NBI (P-NBI) [7]. An ion internal transport barrier (ITB), that is a peaked profile of ion temperature, $T_{i}$, with a steep gradient in the core region, has been formed and the central $T_{i}$ of $6.8 \mathrm{keV}$ has been achieved in a hydrogen plasma with the line-averaged electron density of $2 \times 10^{19} \mathrm{~m}^{-3}$ (see Fig.1).

At the beginning of the shot, the target plasma was heated by the perpendicular NBI alone (P-NBI phase). In this P-NBI phase, the $T_{\mathrm{i}}$ profile is broad and the central $T_{\mathrm{i}}$ is almost 1.5 $\mathrm{keV}$. After the superposition of tangential NBI's with the double power of the P-NBI, the central $T_{\mathrm{i}}$ significantly increases above 5 $\mathrm{keV}$. The electron temperature also increases

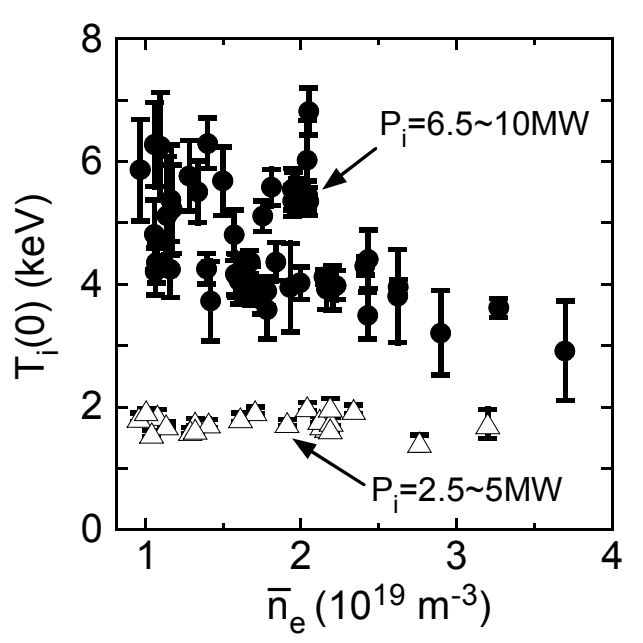

FIG.1 Central ion temperature as a function of line averaged electron density. The maximum values obtained by the combined NBI heating are shown by closed circles. The central ion temperature in the only P-NBI phase is also shown by open triangles. 
up to $4 \mathrm{keV}$, however the electron ITB is not formed simultaneously.

The experimental ion thermal diffusivity significantly decreases to the neoclassical level, indicating a significant reduction of anomalous transport. The analysis of neoclassical ambipolar diffusion [30] showed the enhancement of the negative radial electric field $\left(E_{r}<0\right)$ associated with the $T_{i}$ increase, indicating that the anomalous transport is suppressed by the negative $E_{r}$.

The relation of ion heat flux to the $T_{i}$ gradient was compared between inside $(\rho=0.3)$ and outside $(\rho=0.8)$ of the ITB foot as shown in Fig.2. The quite different dependences of $\chi_{i}$ on the direct ion heating power were observed between inside and outside of the ITB. The dependence of $\chi_{i}$ is fitted as $\chi_{i} \propto\left(P_{i} / n_{i}\right)^{0.59}$ outside of the ITB and $\chi_{i} \propto\left(P_{i} / n_{i}\right)^{-1.61}$ inside of the ITB. Also the temperature dependence of $\chi_{i}$ has been identified as $\chi_{i} \propto T_{i}^{1.10}$ and $\chi_{i} \propto$ $T_{i}^{-0.53}$, outside and inside the ITB, respectively. It is found that the $\chi_{i}$ outside the ITB shows a gyro-Bohm dependence. In contrast, the negative dependence of $\chi_{i}$ on $T_{i}: \partial \chi_{i} / \partial T_{i}<0$ and the heating power: $\partial \chi_{i} / \partial\left(P_{i} / n_{i}\right)<0$ inside the ITB indicate the possibility of further confinement improvement from an increase in heating power.

This new regime accompanies a toroidal rotation driven by tangential NBI and by a spontaneous rotation due to the ion temperature gradient (see Fig.3) [10]. The positive radial electric field drives spontaneous flow in the counter direction at the plasma edge and in the co-direction near the magnetic axis. The difference in the direction of spontaneous flow between the core and edge is considered to be due to a difference in the ratio of the helical ripple to the toroidal ripple. The ion temperature gradient causing the spontaneous toroidal flow

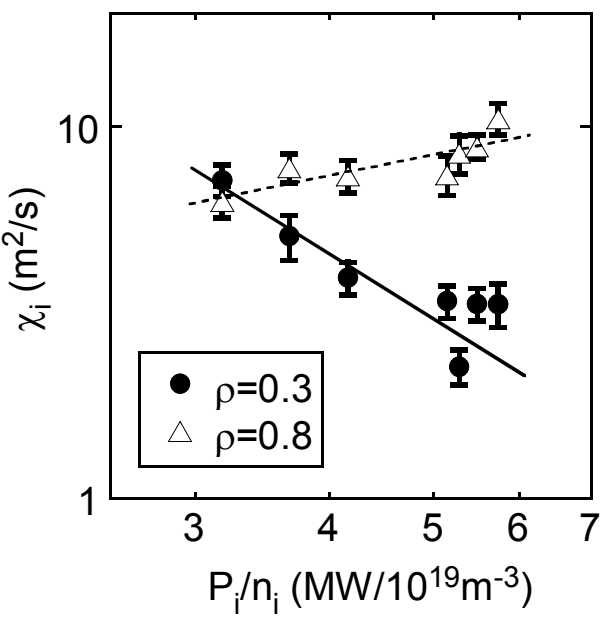

FIG.2 Ion thermal diffusivity $\chi_{i}$ at $\rho=0.3$ (inside the ITB) and 0.8 (outside of the ITB) as a function of direct ion heating power normalized by the ion density. Fitted lines are also shown by a solid line $\left(\chi_{i} \propto\left(P_{i} / n_{i}\right)^{-1.61}\right)$ and broken line $\left(\chi_{i}\right.$ $\left.\propto\left(P_{i} / n_{i}\right)^{0.59}\right)$ for $\rho=0.3$ and $\rho=0.8$, respectively.
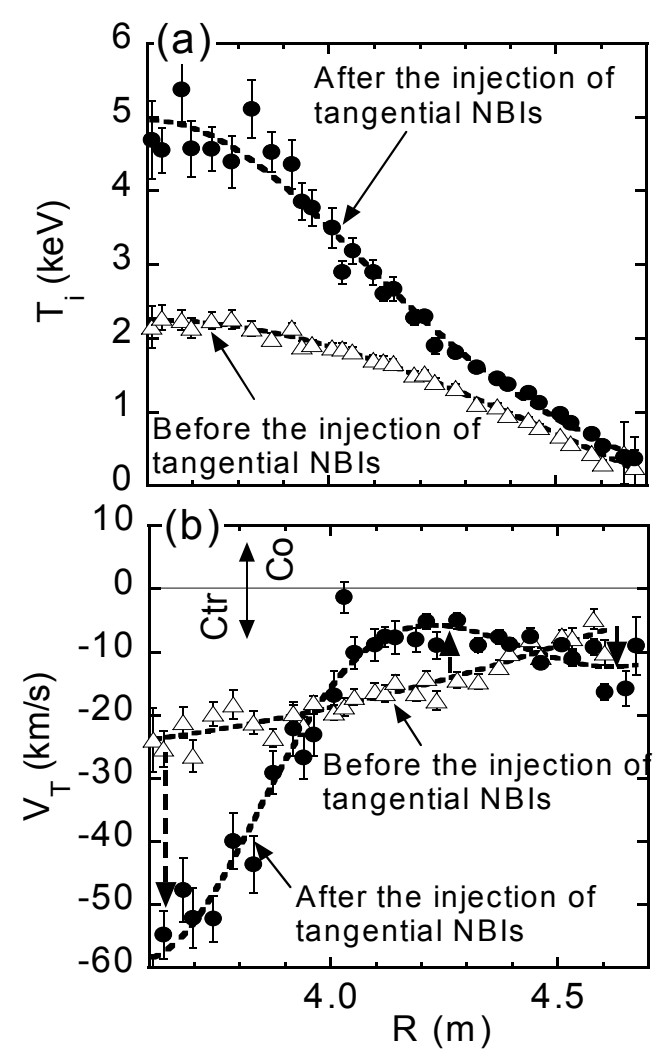

FIG.3 Radial profiles of (a) ion temperature and (b) toroidal flow velocity. Open triangles and closed circles are before and after high-power tangential NBI, respectively. 

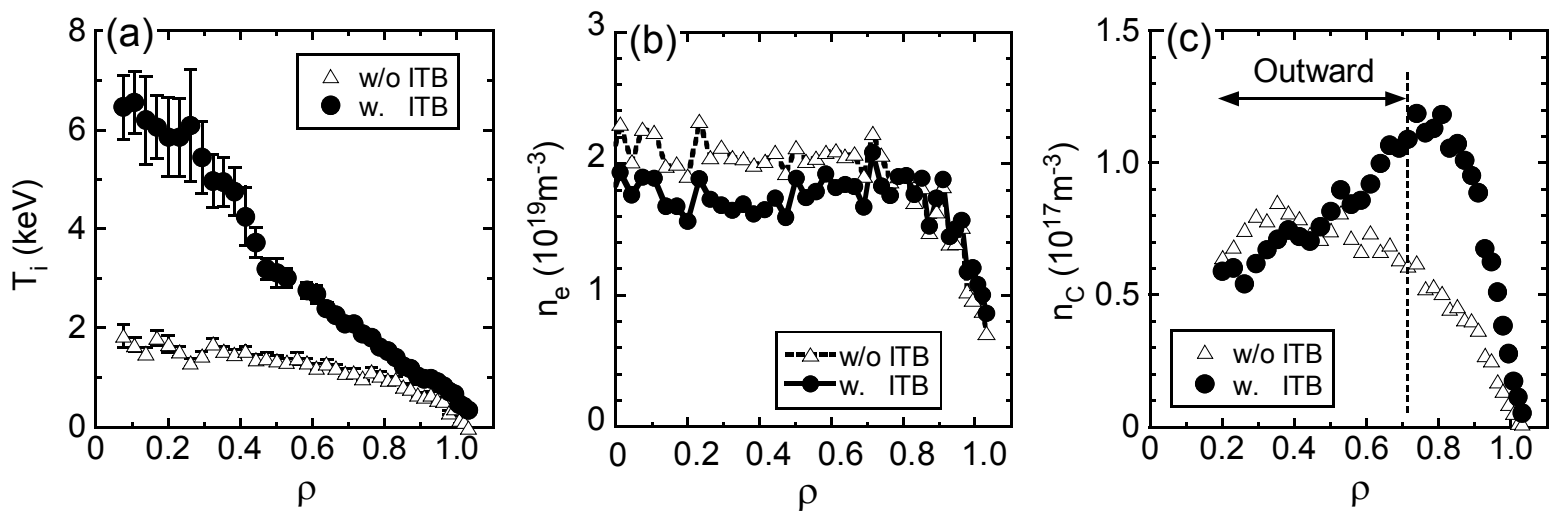

FIG.4 Radial profiles of (a) ion temperature, (b) electron density and (c) carbon density in the phase with (closed circles) and without (open triangles) the ion ITB phase.

is clearly observed near the plasma edge.

Simultaneous achievement of high $T_{i}$ and a low concentration of impurities are crucial for the high fusion triple product, because impurities cause dilution of the fueling particles. Associated with the formation of an ITB, a large outward convection of impurities has been found coincidentally in spite of a negative radial electric field [11]. Since the particle diffusion in the plasma with an ITB is relatively low because of the suppression of the turbulence, the sign of the convection, which appears as an off-diagonal term of the transport matrix, becomes important in the ITB plasma. The radial profiles of carbon density are evaluated from the radial profiles of intensity of the charge exchange line CVI. As seen in Fig.4(c) the carbon density, $n_{C}$, profile changes its shape from a peaked profile to a hollow profile due to an outward convection in the wide region of $\rho=0.25-0.65$ during the ITB phase. In contrast the electron density profile is almost unchanged (see Fig.4(b)) regardless of the significant change in the impurity profile. The radial profile of carbon becomes more hollow as the ion temperature gradient is increased and when the $T_{i}$ gradient is large, an extremely hollow carbon profile "Impurity hole" is observed.

\section{High Beta Regime and MHD instabilities}

The high beta regime has been extended to the diamagnetic beta $\langle\beta\rangle$ of $5 \%$ by optimizing the magnetic configuration. Figure 5 shows the attained beta values as a function of the duration time over which the high beta is held. A quasi-steady state beyond $100 \tau_{\mathrm{E}}$ with $<\beta>>$ $4.5 \%$ has been demonstrated. Pressure driven instabilities observed in LHD are harmless, and the beta value has been successfully increased by enhancement of the heating efficiency.

The spontaneous change of the MHD

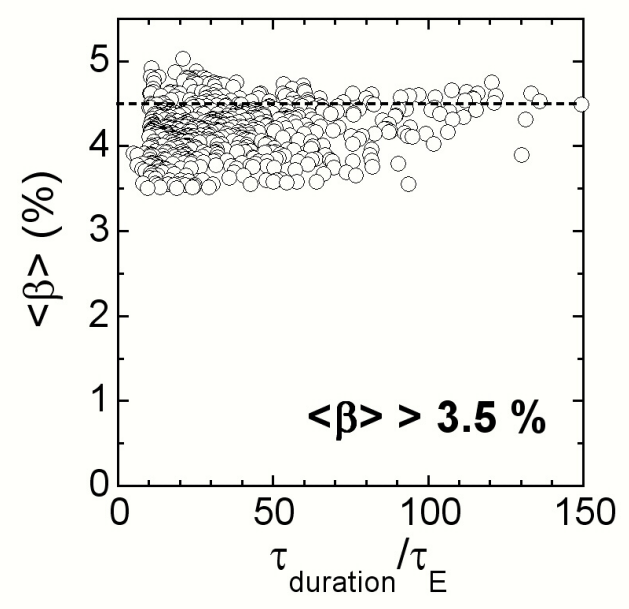

FIG.5 Diamagnetic beta as a function of the duration time of the high beta state normalized by the energy confinement time.The data was selected in the range with $<\beta>>3.5 \%$. 
equilibrium due to finite-beta effects, leading to an extension of the magnetically stochastic region in the periphery and limiting the actual plasma confinement region, is another key issue for realization of the high beta helical reactor. Figure 6 shows the changes of the inner and outer positions of the last closed flux surface (LCFS) and the magnetic axis position as a function of $\langle\beta\rangle$, which was calculated by the 3-D MHD equilibrium code HINT2 [31]. The selected magnetic configuration is the same as that in which the $<\beta>$ of $5 \%$ was obtained in the experiment.

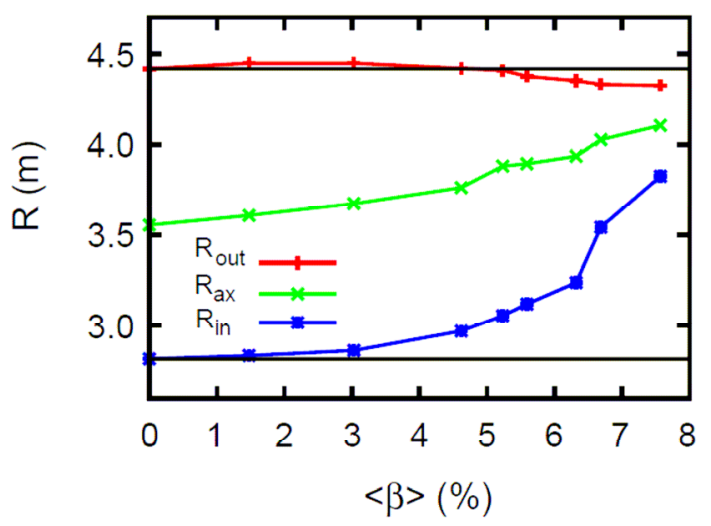

FIG.6 Changes of the inner and outer positions of the LCFS and the magnetic axis position as a function of $\langle\beta\rangle$.

The volume surrounded by the LCFS starts to

shrink when the $\langle\beta>$ exceeds about $3 \%$, and it decreases by $32 \%$ compared to the vacuum case when the $\langle\beta>$ approaches $5 \%$. In contrast, experimental results show that enough temperature gradient still remains outside the calculated LCFS in the highest beta discharge, and the interpretation has been investigated from the viewpoint of transport property in the present high-beta operation and the spontaneous change of magnetic topology. The numerical study suggests that the volume is drastically reduced further for $\langle\beta\rangle>6.7 \%$ because of the loss of the force balance inside the vacuum LCFS.

An IDB scenario, producing peaked pressure profiles by multiple pellet injections, has been also applied to the high beta plasma production and MHD study [8]. The production of the peaked pressure profile leads to the high central beta regime and has an advantage for getting experimental knowledge about the stability and equilibrium beta limits because of the large Shafranov shift and the steep pressure gradient. Figure 7 shows the operation regime in the central beta and the magnetic axis position diagram. The magnetic axis position is a key parameter characterizing MHD equilibrium and stability, and the inward shifted configuration has been selected for standard high-beta operation because of good global energy confinement. In the standard operation with gas-puff or single pellet fueling, the formation of the broad pressure profile allows the access to high-beta regime without core MHD instabilities, while the IDB plasma with peaked pressure profile comes accross them such as sawtooth-like activity and $m=3$ oscillation in the configuration with the inward shifted magnetic axis. In the case with the outward-shifted magnetic axis, operational envelope is restricted by the core density

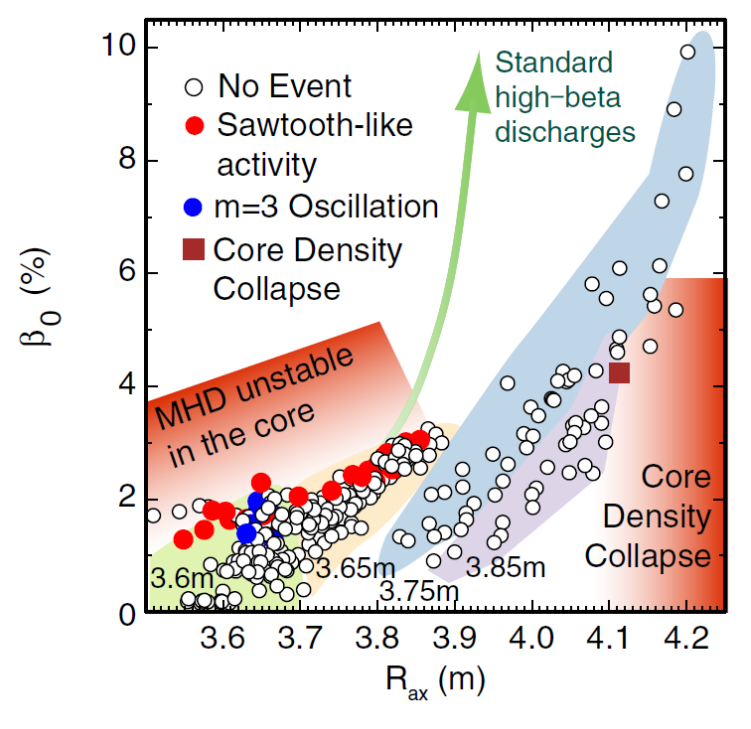

FIG.7 IDB operation regime in the central beta value, $\beta_{0}$, and the magnetic axis position, $R_{a x}$. 
collapse which has a rapid growth time of a few hundred $\mu$ s [13], which will be discussed in the next section. Thus, the best way to produce the high central beta plasmas have been found through the exploration of the magnetic configurations taking into account the Shafranov shift, and a central beta of $10 \%$ has been achieved without serious MHD instability.

A reversed magnetic shear (RS) configuration

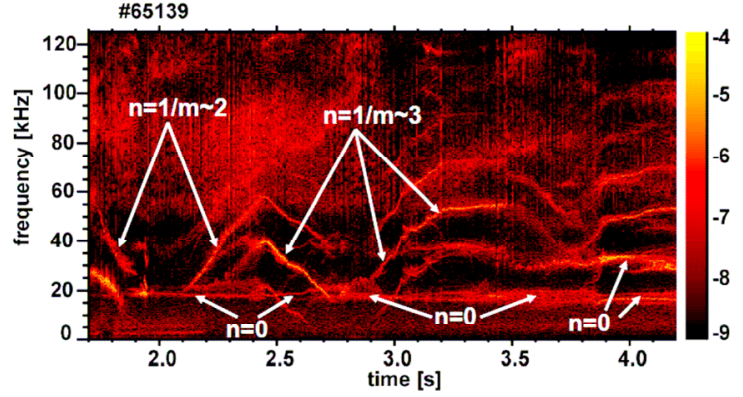

FIG.8 Spectrogram of a magnetic probe signal observed in the reversed shear configuration. having a zero magnetic shear layer away from the magnetic axis was realized by counter neutral beam current drive [21]. When the counter plasma current is rapidly increased in the peripheral region of a high-temperature plasma, it reduces the peripheral rotational transform preferentially and the RS configuration is realized, which was verified by MSE measurements. In such configurations, the $n=1$ reversed shear Alfvén eigenmode (RSAE) and the $n=0$ toroidally symmetric coherent mode, that is, geodesic acoustic mode (GAM) excited by energetic ions were clearly observed in various fluctuation diagnostics, where $n$ is the toroidal mode number. Figure 8 shows a spectrogram of the magnetic probe signal in an example discharge of the RS configuration. The frequency of the $n=1$ RSAE first chirps downward to the minimum of $\sim 18 \mathrm{kHz}$ and then chirps upward. The minimum frequency is close to the GAM frequency. The time evolution of the RSAE frequency is explained very well by ideal MHD theory. In particular, other $n=1$ chirping modes are excited in the phase of $\mathrm{t}>2.4 \mathrm{~s}$ through nonlinear coupling between RSAE and GAM with almost constant frequency of $\sim 18$ $\mathrm{kHz}$. Internal measurements such as electron cyclotron emission (ECE) polychromator and ultra-soft X-ray detector indicate that both RSAE and GAM localize at $r / a=0.3-0.6$ and $r / a=0.1-0.5$, respectively.

\section{High Density Regime with Internal Diffusion Barrier}

The performance of high density discharges with an IDB, initially found with the Local Island Divertor, has been developed in the helical divertor configuration. In contrast with an ITB, the IDB is not accompanied by an improvement in thermal transport as can be seen in Fig. 2 in the relatively flat temperature profile in an IDB region while a temperature gradient is established in the mantle outside of the IDB.

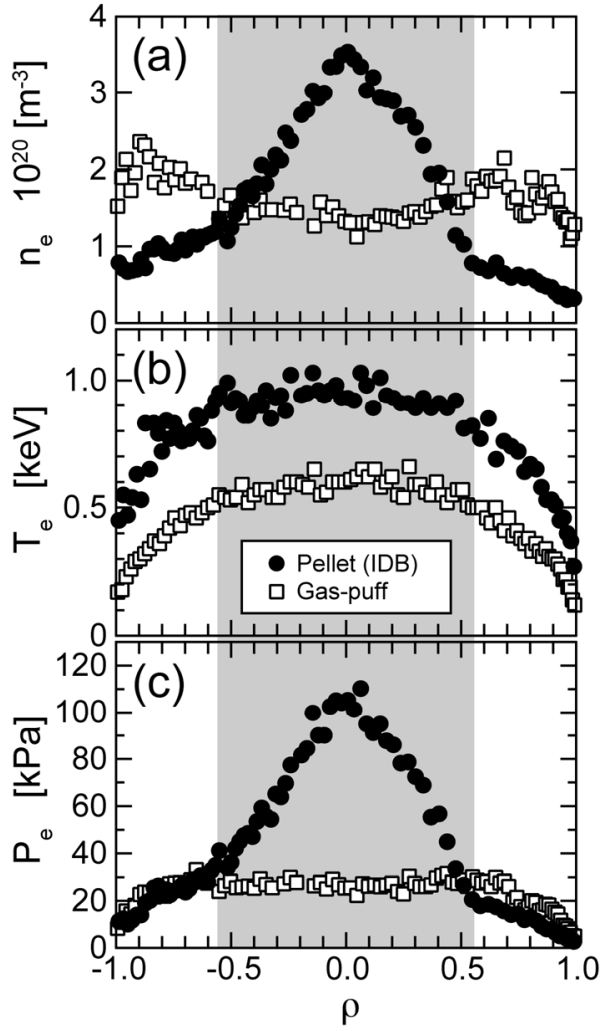

FIG.9 Comparison of (a) electron density, (b) temperature and (c) pressure profiles between pellet and gas-puff discharges. 

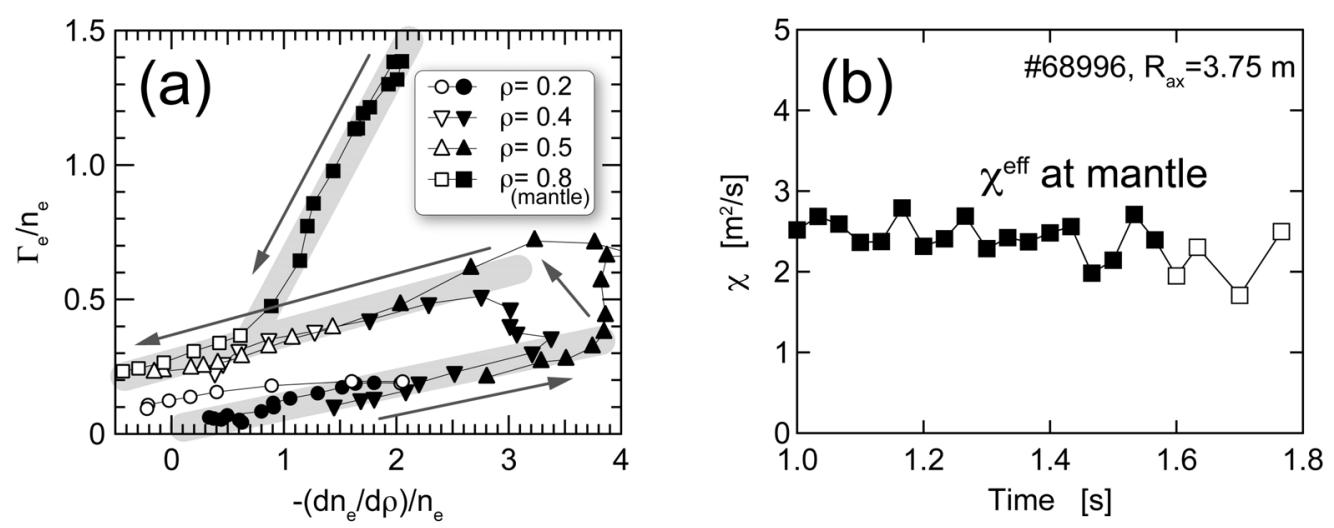

FIG.10 (a) Changes of particle fluxes in different position as a function of density gradient, which are normalized by electron density. (b) Temporal change of thermal diffusivity at $\rho=0.9$. The closed and open symbols denote $w / I D B$ and $w / o$ IDB, respectively.

Typical profiles of the gas-puff and pellet fueled discharges at the same line density $n_{\mathrm{e}} l=$ $3 \times 10^{20} \mathrm{~m}^{-2}$ and same magnetic configuration with $R_{\mathrm{ax}}$ of $3.75 \mathrm{~m}$ are shown in Fig 9 . In the case of pellet fueling, the IDB with a steep density gradient inside $\rho=0.55$ is formed and the central density is remarkably increased while the peripheral density is reduced. The electron temperature of the pellet fueled plasma is higher than that of the gas-puff case despite that the central density is more than double. The plasma pressure profile of the pellet fueled IDB plasma shows an obvious increase of the plasma energy density in the core region and the central pressure is about four times larger than that of the gas-puff fueled plasma.

The characteristics of a particle and a thermal transports in the typical IDB discharge are shown in Fig.10. The data is after pellet fueling, and the IDB phase is shown as a closed symbol. The particle fluxes inside IDB foot $(\rho \leq 0.5)$ gradually increase with an increment of the electron density gradient. The particle diffusivity, $D_{\mathrm{e}}$, which corresponds to the increment rate of the particle flux to the density gradient, is about $0.042 \mathrm{~m}^{2} / \mathrm{s}$ and is almost constant. After the density gradient approaches the maximum, the particle fluxes in the core increase and start to decrease with the reduction of the density gradient and with the outward convection flow. In the reduction phase of the density gradient, the $D_{\mathrm{e}}$ is also constant even in the case of w/o IDB and almost the same as that in the increment phase. The diffusivity at the mantle $(\rho=0.8)$ is relatively large in the IDB phase, whereas it becomes the same as the core one after the IDB formation is broken. The thermal diffusivity at the mantle is almost constant in the discharge and is insensitive to the IDB formation in spite of the significant change of the particle transport. Thus, IDB plasma has two kinds of confinement regions with quite different transport property. The IDB core with the large density gradient has the good particle confinement property, whereas the IDB mantle with the large temperature gradient and with the low density has a tolerance to the radiation collapse, which contributes to support the robustness IDB formation.

The achieved central density becomes higher as the magnetic axis shifts outward and the central density exceeds $1 \times 10^{21} \mathrm{~m}^{-3}$ for $R_{\mathrm{ax}} \geq 3.9 \mathrm{~m}$ at B 2.5 T. The central pressure reaches its largest value in the neighborhood of $R_{\mathrm{ax}}=3.85 \mathrm{~m}$. The maximum central pressure is limited by 


\section{OV/2-4}

the core density collapse [13]. The high density core is expelled on the sub millisecond time scale without having any impact on the central temperature. In order to suppress this unfavorable event, mitigation of Shafranov shift by using ellipticity control (vertical elongation) in a helical device is shown to be effective.

High density operation exposes itself to radiation collapse. Since the operational density limit of helical systems is determined by the loss of power balance, impurity contamination is of much importance. Impurity transport analysis in the ergodic layer of LHD has been carried out using a 3D edge transport code [32]. It indicates that the carbon starts to be screened out from the

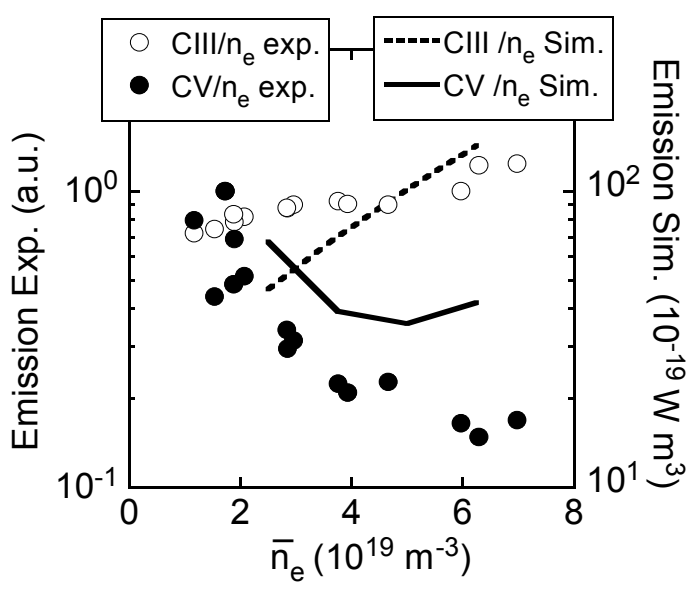

FIG.11 Comparison of CIII emission obtained by VUV monochromators (open circles) and $C V$ with EUV spectrometer (closed circles) with those (CIII in the dotted curve and $C V$ in solid curve) calculated by EMC3-EIRENE, as a function of density. ergodic layer at higher density as the density increases. With increasing density, i.e. collisionality, the friction force on the parallel flow of impurities starts to dominate over the ion thermal force [28]. As a consequence, the impurity is effectively screened in the very edge of the ergodic layer at higher density. As a signature of the screening, emissivity from carbon of different charge states are compared with experiments. Figure 11 shows the carbon radiation intensity of CIII and CV in the experiment, and those by the simulation, as a function of the density. The density dependences are qualitatively in good agreement with each other. It should be noted, that since the ratio of the thermal force and friction force becomes charge independent, the screening model applies also for high-Z impurities such as Fe, another intrinsic impurity in LHD. The mechanism of the screening in the ergodic layer is an important feature of LHD and might be able to explain the tolerance of high density operation like plasmas with an IDB.

\section{Long pulse Operation}

After the achievement of the 54-min. long discharge with a heating power of $490 \mathrm{~kW}$ [4], the increase of the heating power has been an issue in these two years. The steady state heating source is primarily ICRF with the help of $100 \mathrm{~kW}$ of ECH. A scaling relation of the plasma duration time to the applied RF power was derived from accumulated experimental

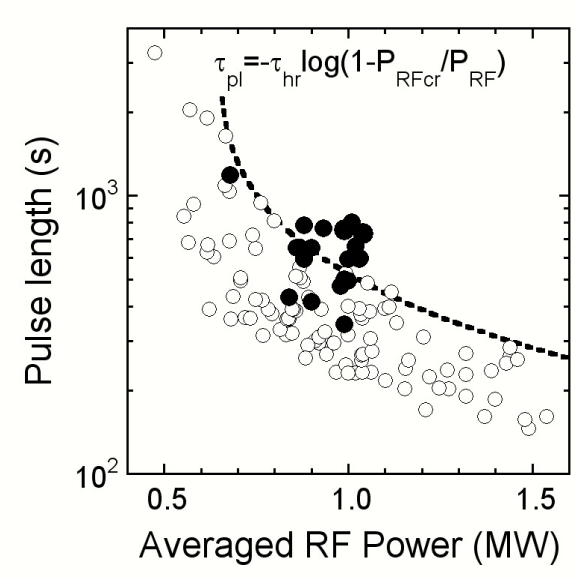

FIG.12 Achieved pulse length of long pulse discharges as a function of $R F$ heating power. Open circles are reported in the last IAEA conference and closed circles have been obtained in these 2 years. Curves are the scaling to evaluate the maximum pulse length. 


\section{$\mathrm{OV} / 2-4$}

data [29]. The experiment data obtained to date are plotted as the pulse length, $\tau_{\mathrm{pl}}$, versus the heating power, $P_{R F}$, in Fig.12. Open circles are reported in the last IAEA conference and closed circles have been obtained in these last 2 years. The relation of the pulse length and the RF power is derived from the envelope of the experimental data (open circles) as an empirical scaling; $\tau_{p l}=-\tau_{h r} \log \left(1-P_{R F c r} / P_{R F}\right)$. Here $P_{R F c r}$ is the critical RF heating power, which is determined by fitting the calculated curve using this scaling. Then $P_{R F c r}$ is determined as 0.65 MW, and $\tau_{h r}$ is the time constant of the heat removal in divertor plates. It indicates that a critical divertor temperature exists. In fact the plasma discharge of about one hour was achieved at $P_{R F} \sim 0.5 \mathrm{MW}$. A new regime of the long pulse was extended over the scaling relation by replacing divertor plates with new ones having better heat conductivity. One of the solid circles shows that a pulse length of $800 \mathrm{~s}$ was achieved with the RF heating power of 1.1 MW. The plasma of $\bar{n}_{e}$ of $6 \times 10^{18} \mathrm{~m}^{-3}$ and $T_{e O}=1.5 \mathrm{keV}$ was sustained with $P_{I C H}=1.0 \mathrm{MW}$ and $P_{E C H}=0.1 \mathrm{MW}$, employing the magnetic axis swing to disperse the plasma heat load to the graphite divertor tiles.

\section{Conclusions}

Remarkable progress in the physical parameters of net-current free plasmas has been made in the LHD in the last two years. The beta value reached $5 \%$ and a high beta state beyond $4.5 \%$ has been maintained for longer than 100 times the energy confinement time. The density and temperature regimes also have been extended. The central density has exceeded $1.0 \times 10^{21} \mathrm{~m}^{-3}$ due to the formation of an IDB. The ion temperature has reached $6.8 \mathrm{keV}$ at the density of 2 $\times 10^{19} \mathrm{~m}^{-3}$, which is associated with confinement improvement which shares commonalities with the ITB. Although plasmas with an IDB and ITB are located in different density regimes, it is found that impurity contamination is significantly reduced for both cases. In a plasma with an IDB, impurity screening due to the friction force exerted by the background plasma flow on impurities suppresses impurity influx in the ergodic layer. The outward convection which cannot be explained by neoclassical theory is pronounced when turbulent driven radial flux is suppressed in a plasma with an ITB.

Control of heat and particles is becoming a critical issue in the high-performance and steady state plasmas reported here. Based on experimental knowledge and performance requirements, the closed helical divertor system which enables much better control of heat and particles is being designed [33].

Experimental observation with detailed diagnostics in LHD promotes theoretical and simulation studies. Validation of the advanced physical models for integrated transport simulation including bootstrap currents [34] and MHD equilibrium/instabilities [35-37], TAE modes [38] and gyro-kinetic simulation of anomalous transport [39] are reported in this conference. Extension of the plasma parameters and the advantage of steady-state operation accelerate the deepening of diversified physical understandings of net current free helical plasmas.

For the next stage of LHD experiments, the constructions of a closed helical divertor and a perpendicular NBI with an accelerating voltage of $80 \mathrm{keV}$ have been started this fiscal year, which will take about four years to complete them. Experiments handling deuterium gas are 


\section{$\mathrm{OV} / 2-4$}

also planned and will begin in a few or several years to achieve final LHD targets.

\section{Acknowledgements}

The authors are grateful to all the technical staff for their excellent works to maintain the LHD. They also acknowledge all contributions from international and domestic collaborators.

\section{References}

[1] A.Iiyoshi et al., Nucl. Fusion 39 (1999) 1245.

[2] T.Mito et al., Fusion Eng. Design 81 (2006) 2389.

[3] S.Murakami et al., Fusion Sci. Technol. 46 (2004) 241.

[4] T.Mutoh et al., Nucl. Fusion 47 (2007) 1250.

[5] S.Imagawa et al., Nucl. Fusion 47 (2007) 353.

[6] O.Motojima et al., Nucl. Fusion 47 (2007) S668.

[7] K.Nagaoka et al., EX/8-2Ra in this conference.

[8] S.Ohdachi et al., EX/8-1Rb in this conference.

[9] R.Sakamoto et al., EX/8-1Ra in this conference.

[10] M.Yoshinuma et al., EX/P5-1 in this conference.

[11] K.Ida et al., EX/8-2Rb in this conference.

[12] N.Ohyabu et al., Phys. Rev. Lett. 97 (2006) 055002.

[13] H.Yamada et al, Plasma Phys. Control. Fusion 49 (2007) B487.

[14] T.Fukuda et al., EX/P5-11 in this conference.

[15] K.Tanaka et al., EX/P5-6 in this conference.

[16] S.Inagaki et al., EX/P5-10 in this conference.

[17] H.Igami et al., EX/P6-13 in this conference.

[18] S.Kubo et al., EX/P6-14 in this conference.

[19] H.Kasahara et al., EX/P6-30 in this conference.

[20] S.Murakami et al., EX/P8-3 in this conference.

[21] K.Toi et al., EX/P8-4 in this conference.

[22] M.Nishiura et al., EX/P8-5 in this conference.

[23] T.Hino et al., EX/P4-8 in this conference.

[24] N.Ashikawa et al., EX/P4-7 in this conference.

[25] N.Yoshida et al., FT/2-1 in this conference.

[26] Y. Takeiri et al., Nucl. Fusion 47 (2007) 1078.

[27] K.Y.Watanabe et al., Nucl. Fusion 45 (2005) 1247.

[28] M.Kobayashi et al., EX/P9-4 in this conference.

[29] R.Kumazawa et al., EX/P6-29 in this conference.

[30] M.Yokoyama et al., Phys. Plasmas 15 (2008) 05611.

[31] Y.Suzuki et al., TH/P9-19 in this conference.

[32] M.Kobayashi et al., Contrib. Plasma Phys. 48 (2008) 255.

[33] S.Masuzaki et al., EX/P4-24 in this conference.

[34] N.Mizuguchi et al., TH/P9-17 in this conference.

[35] Y.Nakamura et al., EX/P6-20 in this conference.

[36] M.Sato et al., TH/P9-18 in this conference.

[37] H.Miura et al., TH/P9-16 in this conference.

[38] Y.Todo et al., TH/P3-9 in this conference.

[39] T.H.Watanabe et al., TH/P8-20 in this conference. 